\title{
The Location of Cues Promoting Selective Reinnervation of Axolotl Muscles
}

\author{
Donald J. Wigston ${ }^{1}$ and Sean P. Donahue ${ }^{2}$ \\ Departments of ${ }^{P}$ Physiology and ${ }^{2}$ Anatomy and Cell Biology, Emory University School of Medicine, Atlanta, Georgia 30322
}

The selective reinnervation of muscles suggests that muscles have intrinsic recognition cues that promote selective synaptogenesis. For example, the anterior and posterior heads of the axolotl iliotibialis (ILT) muscle are preferentially reinnervated by their original motoneurons even after surgically exchanging them. The nature and location of cues that promote such selectivity are unknown, although previous work suggests that the muscle fibers themselves might harbor the relevant molecules. To address this question, we removed anterior and posterior ILT muscles, destroyed their myofibers by surgically damaging them and treating them with bupivicaine, and reimplanted them in either a normal or a reversed anterior/posterior orientation. After the regenerated myofibers became innervated, we stimulated different spinal nerves and recorded the synaptic potentials evoked in muscle fibers. Our results showed that if the muscles were removed, damaged, and reimplanted in their original positions, the segmental origin of inputs to the regenerated myofibers was similar to that seen in normal muscles and in muscles reimplanted with their myofibers intact. However, muscles that were removed and damaged but regenerated in new positions were innervated differently from normal muscles and from muscles whose myofibers survived transplantation. Thus, the site at which a muscle regenerates has an influence on the source of the muscle's reinnervation. Nevertheless, the innervation of muscles that regenerated after transplantation to a foreign site was not strictly appropriate for the new position, but was biased towards the muscle's original innervation pattern. Therefore, some, but not all, of the cues that reflect the original identity of the transplanted muscles survive the replacement of its myofibers.

The consistent patterns of synaptic connectivity seen in the nervous systems of vertebrates suggest that the assembly and interconnection of neuronal systems occur within constraints that favor the formation of specific connections. Events that influence how neurons become interconnected probably operate at several different levels: the generation and survival of the right numbers and kinds of neurons; migration of neurons to appropriate locations; the growth and guidance of axons into the general regions of their future target cells; selective synaptogenesis with particular target cells; and the elimination of some

\footnotetext{
Received Oct. 26, 1987; revised Jan. 11, 1988; accepted Jan. 21, 1988.

We wish to thank P. Shuler and A. McLean for assistance, and V. Boss, L Crews, A. English, P. Lennard, D. Purves, and J. Sanes for helpful suggestions. Supported by a grant from the NIH.

Correspondence should be addressed to D. J. Wigston.

Copyright (C) 1988 Society for Neuroscience $0270-6474 / 88 / 093451-08 \$ 02.00 / 0$
}

of the initial axonal projections and synapses. All of these may be important in establishing the patterns of connectivity seen in the adult nervous system.

The neuromuscular system is a convenient place to study neuronal specificity because of its accessibility and relative simplicity. It has been shown that the patterns of axonal projection from spinal motoneurons to limb muscles are not produced by the selective death of neurons that make inappropriate connections (Landmesser, 1978; Oppenheim, 1981; Lance-Jones, 1982; Farel and Bemelmans, 1985; but compare Lamb, 1976), unlike in some purely neuronal systems (Clarke and Cowan, 1976; O'Leary and Cowan, 1982; O'Leary et al., 1986). On the other hand, the guidance of axons towards particular target muscles surely plays an active role in establishing normal connectivity; motoneurons displaced from their usual positions can still successfully navigate to their proper muscle (Lance-Jones and Landmesser, 1980, 1981). The selective elimination of inappropriately positioned neuromuscular connections may further sharpen the topographic mapping of motoneuron pools onto their muscles (McGrath and Bennett, 1979; Brown and Booth, 1983; Bennett and Lavidis, 1984; but see English, 1986; Donahue and English, 1987). Although many studies of neuromuscular systems have examined aspects of neuronal death, axonal navigation, and both axon and synapse elimination, much less is known about the selectivity of synapse formation. For this reason, our goal has been to establish a muscle system in which selective synapse formation can be shown to occur, in the hope that the simplicity and accessibility of this system will help in further understanding the mechanisms responsible for synaptic selectivity. The hindlimb muscles of the axolotl satisfy this requirement.

Urodele amphibians can recover normal use of their limbs after denervation (Weiss, 1936). This may be achieved, at least in part, by selective reinnervation of individual muscles (Stephenson, 1979; Wigston, 1986). For example, when the anterior and posterior iliotibialis (ILT) muscles of the hindlimb are surgically exchanged, each muscle is preferentially reinnervated by its original motoneurons (Wigston and Kennedy, 1987). This suggests that regenerating axons can distinguish between, or be distinguished by, the anterior and posterior ILT. The studies we report here were aimed at exploring the location and source of cucs that might distinguish muscles from one another or facilitate their recognition of particular axons. Our approach was to eliminate a muscle's myofibers selectively, components likely to bear or produce cues used in selective reinnervation. Our results indicate that muscle recognition cues are not restricted to myofibers, but may be shared with other muscle cell types or the extracellular matrix. Preliminary accounts have 
Figure 1. Dorsal surface of left and right hindlimbs of the axolotl, illustrating muscle treatment and reimplantation. The 4 proximal extensor muscles outlined are the puboischio femoralis internus, anterior iliotibialis, posterior iliotibialis, and iliofibularis. On one side (see right), the anterior and posterior ILT was removed and pinned in a Sylgard-lined dish filled with bupivicaine $(0.75 \%)$, cut transversely in 2 places, leaving a thin strand of intact fibers along the edges, and left in bupivicaine for $1 \mathrm{hr}$ before being sutured back into the original site. On the contralateral side (see left), the muscles were simply removed and thereby denervated, and immediately returned to their former position.

appeared elsewhere (Donahue and Wigston, 1987; Wigston and Donahue, 1987).

\section{Materials and Methods}

Antmals. We used male and female wild-type axolotls $(16-20 \mathrm{~cm})$, obtained from Central Valley Biological (Gardnerville, NV) or Indiana University. They were housed individually in $20 \%$ modified Holtfreter's solution and fed twice weekly.

Surgery. Animals were randomly divided into 2 groups and anesthetized by immersion in $0.1 \%$ MS222 (Tricaine methanesulfonate). In the first $(n=12)$, we bilaterally removed the anterior and posterior ILT muscle, thereby severing its nerves, and immediately reimplanted the entire ILT from one side in its former site. On the other side, we pinned the 2 heads of ILT in a Sylgard-lined dish, cut their fibers transversely at 2 different proximodistal levels so as to leave thin strands of intact fibers at both edges (see Fig. 1), and soaked them in bupivicaine $(0.75 \%)$ for $1 \mathrm{hr}$. This procedure killed virtually all myofibers in the muscle, but did not prevent their regeneration. We varied the side chosen for treatment and coded the animals so that at the time of recording, the experimenter was unaware of which side had been treated.

A second group of axolotls $(n=11)$ underwent surgical transplantation of the right and left ILT muscles, as described previously (Wigston, 1986; Wigston and Kennedy, 1987), but with some modifications. Briefly, we removed the anterior and posterior heads of ILT bilaterally, pinned them in a dish of bupivicaine and damaged them by cutting their fibers transversely, as above. After $1 \mathrm{hr}$ we sutured the left anterior and posterior ILT into the right hindlimb, and vice versa. In both limbs, the contralateral anterior ILT then occupied the former position of the posterior ILT, whereas the contralateral posterior ILT replaced the original anterior ILT (Fig. 2).
Electrophysiological recording. Some 2-4 months after surgery, we removed both left and right hindlimbs for in vitro recording, as described previously (Wigston, 1986). We impaled muscle fibers in both anterior and posterior ILT and accepted the first 10-12 impalements in each head that produced a stable baseline, reasonable noise level, and resting potential of $\geqq-60 \mathrm{mV}$. We were careful to sample fibers across the full width and depth of each muscle. We noted the segmental origin of synaptic potentials evoked in each fiber by stimulating spinal nerves 15-18 in turn and varying the stimulus intensity to test for multiple inputs. We represented the innervation of each muscle with a segmentally weighted average of all the inputs we recorded in that muscle: for example, if we recorded a total of 16 inputs (one from spinal nerve 15 ; 5 from nerve $16 ; 8$ from nerve 17 ; and 2 from nerve 18 ), we would assign that muscle a value of $[(1 \times 15)+(5 \times 16)+(8 \times 17)+(2 \times$ $18)] /(1+5+8+2)=267 / 16)=16.69$. The larger this value, the more caudal the spinal origin of the muscle's innervation (Wigston and Sanes, 1985; for details, see Wigston, 1986). Finally, for each limb in which we obtained 10 or more successful impalements in each head of ILT (9/46 limbs were rejected), the value obtained for the original anterior IL'I was subtracted from that for the original posterior ILT to yield the difference in average segmental innervation of the 2 muscles. Differences between means were evaluated using Student's $t$ test.

Histology. Eight additional animals were decapitated at various times following bilateral muscle treatment and reimplantation, and the muscles examined to determine the time course and extent of myofiber degeneration and regeneration (Fig. 3). Muscles were removed and fixed in HEPES-buffered $2 \%$ glutaraldehyde $(110 \mathrm{~mm} \mathrm{NaCl}, \mathrm{pH}, 7.2)$, dehydrated, embedded in paraffin, and sectioned at $10 \mu \mathrm{m}$. Sections were then stained with toluidine blue to reveal muscle fibers, examined by light microscopy, and photographed with Kodak Technical Pan film. Fifteen muscles used for electrophysiology were prepared in a similar
Figure 2. Treatment and transplantation of axolotl ILT muscles. Anterior and posterior ILT muscles were removed bilaterally, immersed in bupivicaine, and cut transversely in 2 places, leaving a narrow strip of intact fibers along each edge. After $1 \mathrm{hr}$ they were sutured into the contralateral limb so that the anterior ILT occupied the position vacated by the contralateral posterior ILT and vice versa.

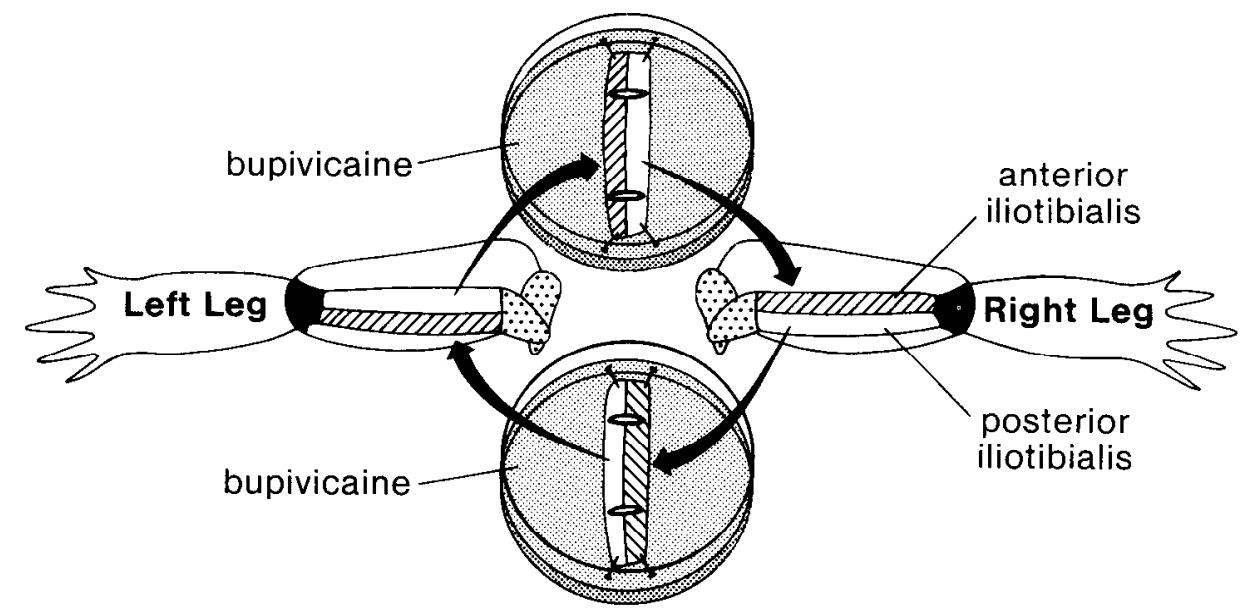



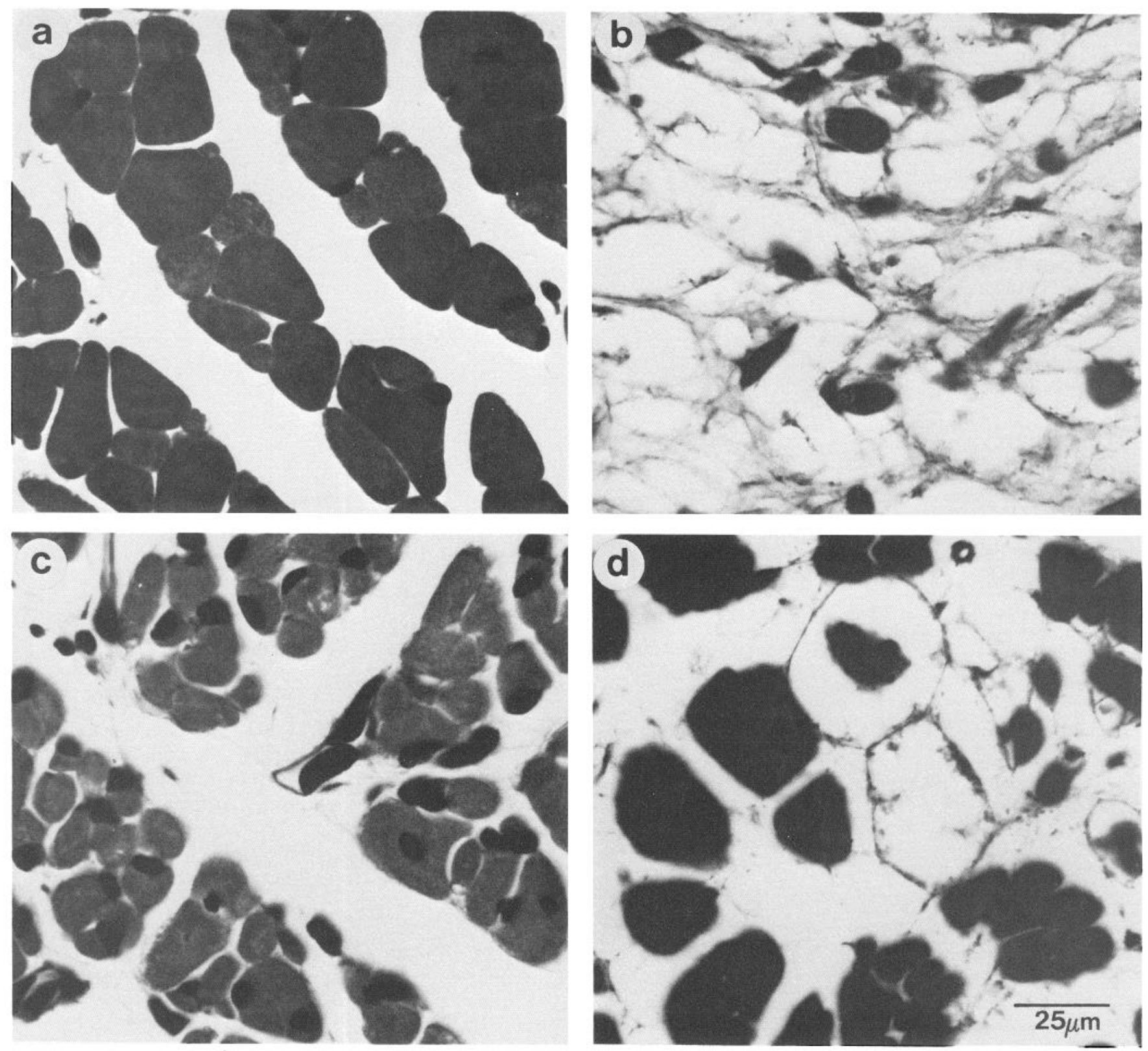

Figure 3. Photomicrographs illustrating the effects of surgical damage and bupivicaine treatment on axolotl ILT muscles. $a$, Normal muscle stained with toluidine blue. $b$, Day 10 following treatment and reimplantation; all myofibers have degenerated except for narrow columns at the anterior and posterior margins of the combined ILT (not shown). The darkly staining profiles are probably macrophages. $c$, Day 40 following treatment and reimplantation; many muscle fibers have regenerated. Some fibers are already of normal size; most, however, are smaller than normal. Centrally located nuclei, characteristic of regenerating myofibers (Benoit and Belt, 1970), are common. $d$, Day 70 after treatment and reimplantation. Myofibers have regained normal size. Regeneration is not complete, however; some empty and partially filled basal lamina sheaths can still be found. $b$ and $d$, Deliberately overexposed to reveal the lightly stained extracellular matrix. Magnification is the same for $a-d$.

manner after recording, to observe the extent of muscle fiber regeneration at longer times after treatment.

\section{Results}

\section{Degeneration, regeneration, and reinnervation of axolotl} myofibers

Mammalian muscle fibers are quickly destroyed by soaking them briefly in bupivicaine (Benoit and Belt, 1970). Since we found that axolotl myofibers were not all killed with this myotoxin, even after soaking them for up to $1 \mathrm{hr}$, we treated the muscles with bupivicaine and also damaged them by cutting their my- ofibers, as described in Materials and Methods and shown in Figures 1 and 2. This caused an almost total loss of myofibers within about $10 \mathrm{~d}$ (Fig. $3 \mathrm{~b}$ ), except for a narrow column of surviving fibers along the 2 outer edges of the ILT muscle. We therefore deliberately avoided muscle fibers along the outer edges of the muscles in our recording experiments. By $30-40 \mathrm{~d}$ after this treatment, many small fibers had regenerated throughout the muscles (Fig. 3c), probably from satellite cells associated with each myofiber (Mauro, 1961). By $70 \mathrm{~d}$ after treatment (Fig. $3 d$ ), the myofibers had grown substantially and reinnervated fibers were encountered often. We recorded a total of 1133 

innervating axolotl iliotibialis muscles. Percentage of all inputs from spinal nerves 15-18 recorded intracellularly from upper left: anterior ILT muscles that were reimplanted in their original site with myofibers intact. Lower left, undamaged posterior ILT muscles that were reimplanted in their former site. Upper middle, anterior ILT muscles that regenerated and were reinnervated in their original site. Lower middle, posterior ILT muscles that regenerated and were reinnervated in their usual position. Upper right, anterior ILT muscles that regenerated and were reinnervated after transplantation to the site of the contralateral posterior IL'I. Lower right, posterior ILT muscles that regenerated and were reinnervated in the former site of the contralateral anterior ILT.
Figure 4. Segmental origin of axons

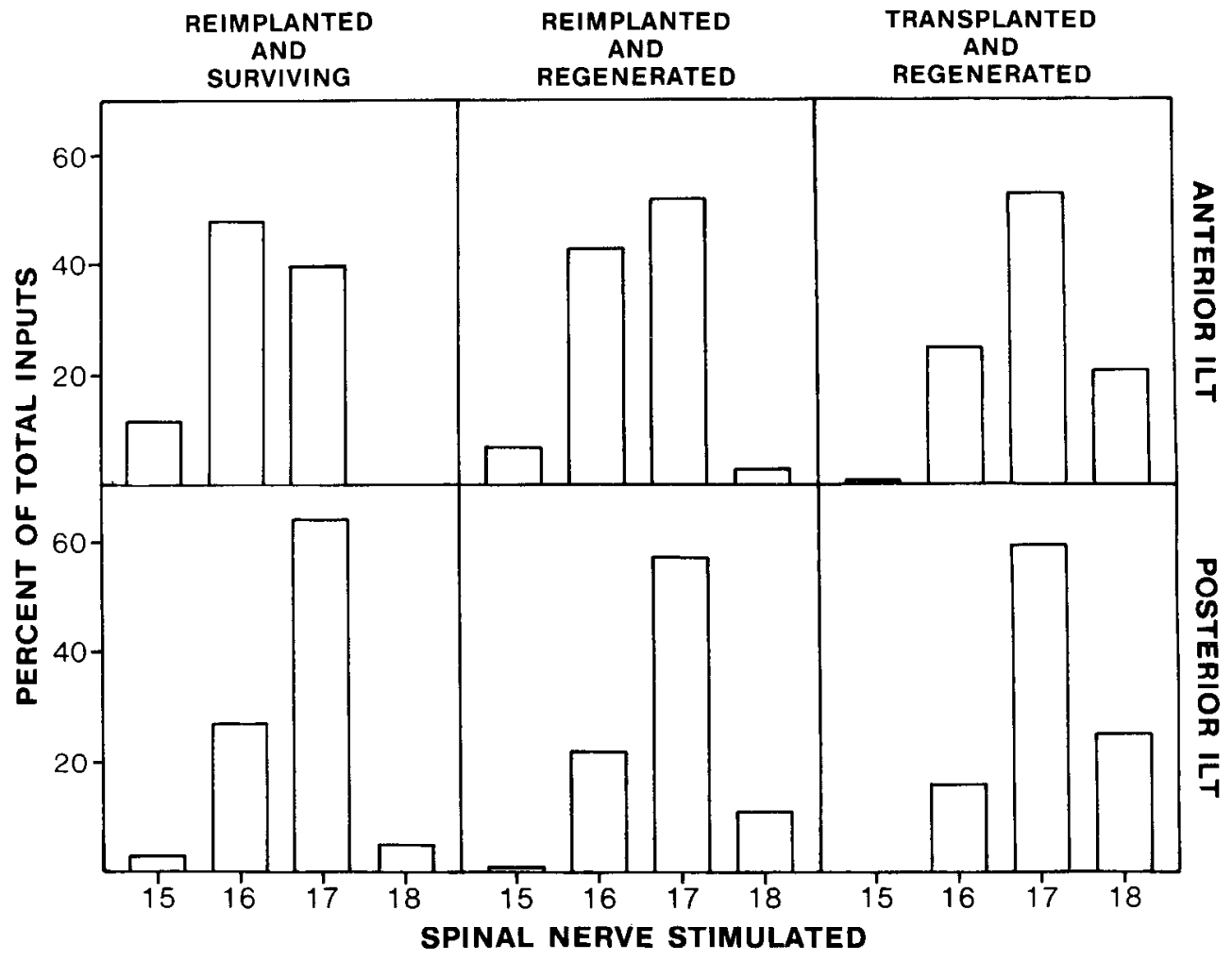

inputs in 687 innervated fibers 69-112 d after degeneration and regeneration (see Table 1). This corresponds to an avcrage of about 1.6 inputs/innervated fiber, and was equal to the density of innervation that we observed in muscles that were reimplanted intact, and similar to that seen in normal, unoperated muscles (Wigston, 1986).

\section{Reinnervation of myofibers regenerating in their original site}

To test whether a muscle's original myofibers are required for selective reinnervation, we removed the anterior and posterior ILT muscle bilaterally in 12 axolotls. On one side we immediately reimplanted the ILT into its former site, while on the other we damaged the ILT muscle and soaked it in bupivicaine for $1 \mathrm{hr}$ to destroy its original myofibers. We then reimplanted the muscles as closely to their original sites as possible. After $71-98 \mathrm{~d}$, by which time the new myofibers were reinnervated, we impaled 10-12 muscle fibers in each head, and noted the segmental origin of synaptic potentials evoked by stimulating spinal nerves 15-18 in turn (Fig. 4). We represented the source of the motor axons reinnervating each muscle by calculating an average of all the inputs recorded in a particular muscle head, weighted according to the segment of their origin (see Materials and Methods; Wigston and Sanes, 1982, 1985; Wigston, 1986). The values obtained for each anterior and posterior ILT muscle are shown in Figures 5 and 6; the higher these values, the more caudal the spinal origin of a muscle's innervation. We then subtracted this weighted average calculated for the anterior ILT from that of the corresponding posterior ILT, to obtain a value representing the difference between the sources of each muscle's innervation. This approach effectively eliminates differences between animals in the rostrocaudal position of motoneurons innervating the hindlimb. The mean difference between the average segmental innervation of 12 anterior/posterior ILT muscle pairs that regenerated after reimplantation in their original site $(0.45 \pm 0.12$; mean \pm SEM; Fig. 8 , column 2$)$ did not deviate significantly from the mean difference between the average seg-

Table 1. Reinnervation of axolotl iliotibialis muscles

\begin{tabular}{|c|c|c|c|c|c|c|c|c|}
\hline & \multicolumn{4}{|c|}{ Surviving } & \multicolumn{4}{|c|}{ Regenerating } \\
\hline & \multicolumn{2}{|c|}{ Reimplanted in situ } & \multicolumn{2}{|c|}{ Transplanted $^{a}$} & \multicolumn{2}{|c|}{ Reimplanted in situ } & \multicolumn{2}{|c|}{ Transplanted } \\
\hline & Ant. & Post. & Ant. & Post. & Ant. & Post. & Ant. & Post. \\
\hline Innervated fibers studied & 124 & 127 & 431 & 396 & 129 & 134 & 211 & 213 \\
\hline Total inputs & 184 & 209 & 789 & 698 & 185 & 231 & 367 & 350 \\
\hline \multirow[t]{2}{*}{ Average segmental innervation ${ }^{b}$} & 16.30 & 16.72 & 16.61 & 16.94 & 16.42 & 16.87 & 16.90 & 16.98 \\
\hline & \pm 0.18 & \pm 0.14 & \pm 0.06 & \pm 0.06 & \pm 0.16 & \pm 0.13 & \pm 0.15 & \pm 0.14 \\
\hline Number of muscles: & (11) & (11) & $(37)$ & (32) & $(12)$ & $(12)$ & $(16)$ & $(16)$ \\
\hline
\end{tabular}

${ }^{a}$ From Table 2 (long-term) in Wigston (1986).

${ }^{b}$ Calculated by computing the mean of the average segmental innervation of each muscle in each group. 


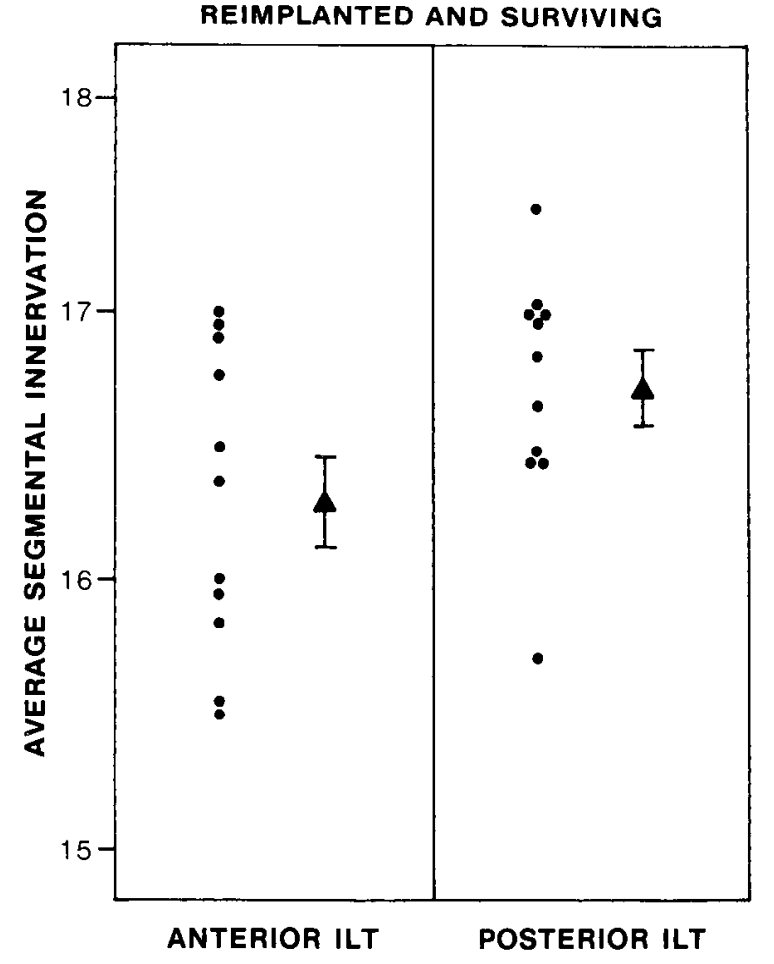

Figure 5. Average segmental innervation of all reimplanted, surviving anterior (left) and posterior (right) ILT muscles. Each dot represents one anterior or posterior ILT muscle. The greater the value of the average segmental innervation, the more caudal the segmental origin of the muscle's innervation. Only those muscles that met the criteria described in Materials and Methods are included. Triangles and error bars, means \pm SEM.

mental innervation of 11 anterior/posterior ILT muscle pairs that were reimplanted in their original site with myofibers intact $(0.41 \pm 0.14$; Fig. 8, column 1) or that of normal muscles (Wigston, 1986). The fidelity of reinnervation of muscles that regenerate and are reinnervated in situ shows that reinnervation in the normal pattern can occur even in the absence of cues associated directly with the original myofibers.

\section{Reinnervation of myofibers regenerating in a new site}

To test whether muscles that regenerate in a new site can be reinnervated selectively, we removed the anterior and posterior ILT bilaterally in 11 axolotls, and treated them as above to kill their myofibers. We then sutured the damaged muscle into the contralateral limb, so that the positions of the anterior and posterior heads were reversed (Fig. 2). After 69-112 d, we recorded synaptic potentials evoked in individual myofibers in vitro, noted their segmental origin (Fig. 4), and determined the average segmental innervation of each head (Fig. 7). For 14 ILT muscles in which we recorded from at least 10 fibers in each head, the difference in segmental innervation between the anterior and posterior ILT was $0.08 \pm 0.12$. This value was significantly different from that obtained for surviving anterior and posterior ILT muscles transplanted in a similar fashion $(0.35$ $\pm 0.05 ; n=30, p<0.025$; Wigston, 1986; see Fig. 8, column 3 ). The lower value for the average difference in segmental innervation after myofiber regeneration indicates a reduced selectivity in the reinnervation of regenerated anterior and posterior ILT, and suggests that information associated with a muscle's original myofibers must contribute to selective reinnervation. Our results indicate, however, that this information

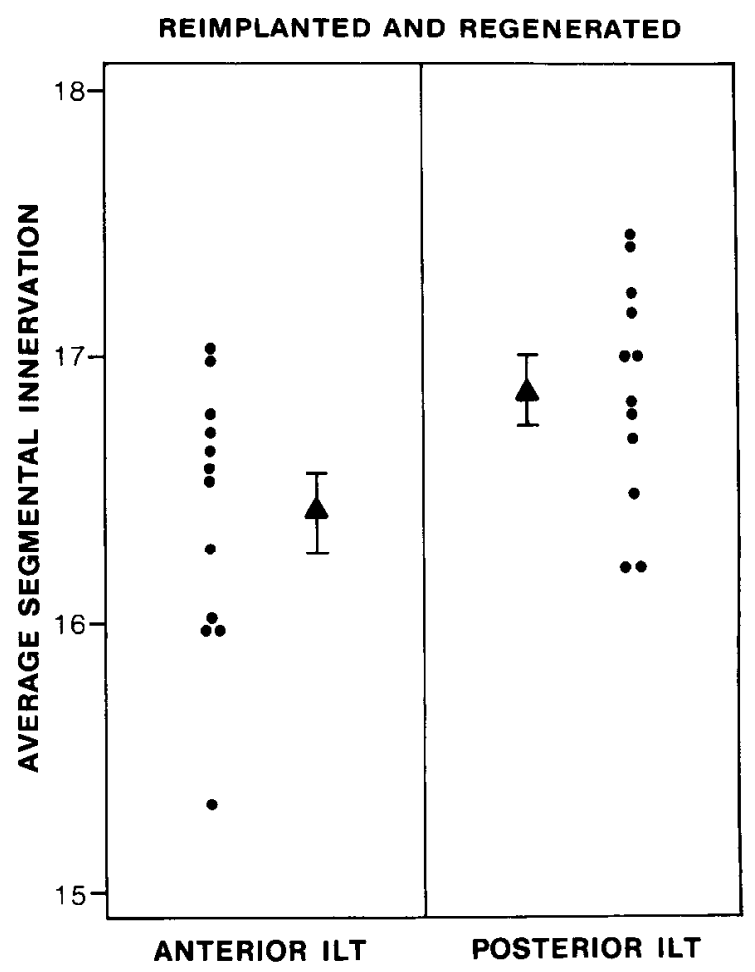

Figure 6. Average segmental innervation of all muscles that regenerated in their original site. Left, Anterior muscles. Right, Posterior muscles. Dots represent individual muscles; triangles represent means \pm SEM.

must also be available at other sites within the muscle (see below).

\section{Discussion}

Several different experiments with both mammalian (Wigston and Sanes, 1982, 1985) and axolotl (Holder et al., 1982; Wigston, 1986; Wigston and Kennedy, 1987) muscle suggest the existence of cues that promote the selective reinnervation of adult muscle. Although we imagine that the selective reinnervation of muscles must be mediated by a system of molecules or molecular complexes, we have at present no way of studying these molecules directly. We therefore attempted to learn where cues that mediate selective reinnervation are located, in the hope that this might help in their subsequent identification.

Previous work with rat intercostal muscles indicated that the cues might be associated exclusively with a muscle's original myofibers: after regeneration of their myofibers, no selectivity was seen in the reinnervation of intercostal muscles transplanted to the neck and reinnervated by autonomic preganglionic axons (Wigston and Sanes, 1985). Our approach, therefore, was to destroy a muscle's original muscle fibers and thus any selectivity cues that might be present on or within them. Since new myofibers quickly regenerated, probably from undifferentiated satellite cells (Mauro, 1961), and were reinnervated, we could use conventional intracellular recording to determine the segmental origin of axons reinnervating muscles that regenerated after being transplanted to new locations.

Our results indicate that when muscles are not moved from their native site, replacement of their original myofibers by regeneration does not produce an observable change in the segmental origin of the motor innervation they subsequently receive. Hence, muscles can be reinnervated normally, even in 
TRANSPLANTED AND REGENERATED

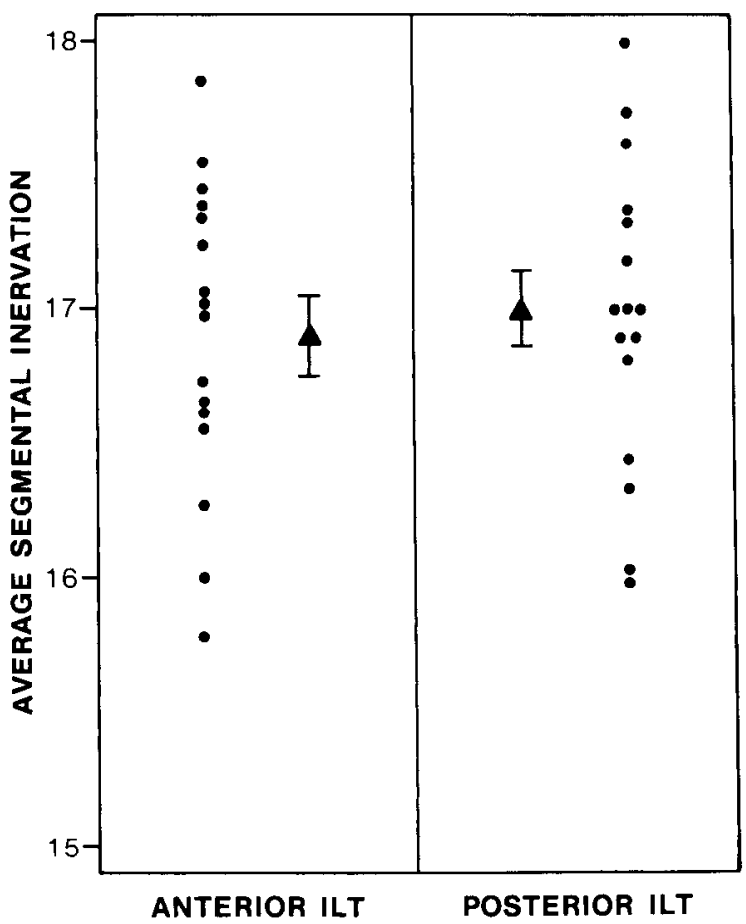

Figure 7. Average segmental innervation of muscles that regenerated in a novel site. Left, Anterior muscles. Right, Posterior muscles. Dots represent individual muscles; triangles, means and their SEs. Some muscles have been excluded (see Materials and Methods).

the absence of their original fibers. However, when damaged muscles generate a new complement of muscle fibers after transplantation to a new site, they are reinnervated differently from normal: the fidelity of reinnervation is considerably less than that seen in muscles that regenerate without transplantation or in muscles that have been transplanted with their myofibers intact (see Fig. 8). Barring an unknown side effect of bupivicaine treatment, this suggests that at least some selectivity cues are associated with a muscle's original myofibers and are irrevocably lost or modified when the original myofibers are replaced. In addition, it suggests that, in the absence of muscle-specific cues, reinnervation would be determined by the site in which a muscle is implanted.

The anatomical arrangement of the nerves that supply anterior and posterior ILT in the axolotl is such that both heads are easily accessible to the branches that previously innervated each muscle. It is possible that regenerating axons might initially establish synapses nonselectively with either muscle, and that their arborizations are subsequently refined by pruning or synapse elimination. Previous results, howcver, suggest that synapses are reestablished selectively from the outset, or at least by the time they can be detected physiologically (Wigston, 1986), and that no axons innervate both muscles simultaneously (Wigston and Kennedy, 1987). Thus, regenerating axons appear to restrict their functional synapses to a single head of ILT, although more detailed studies would be required to determine the precise nature of the growth and branching patterns of individual axons.

In contrast to the results reported here, Wigston and Sanes (1985) found that intercostal muscles that regenerated after bupivicaine treatment were reinnervated nonselectively by the pre-

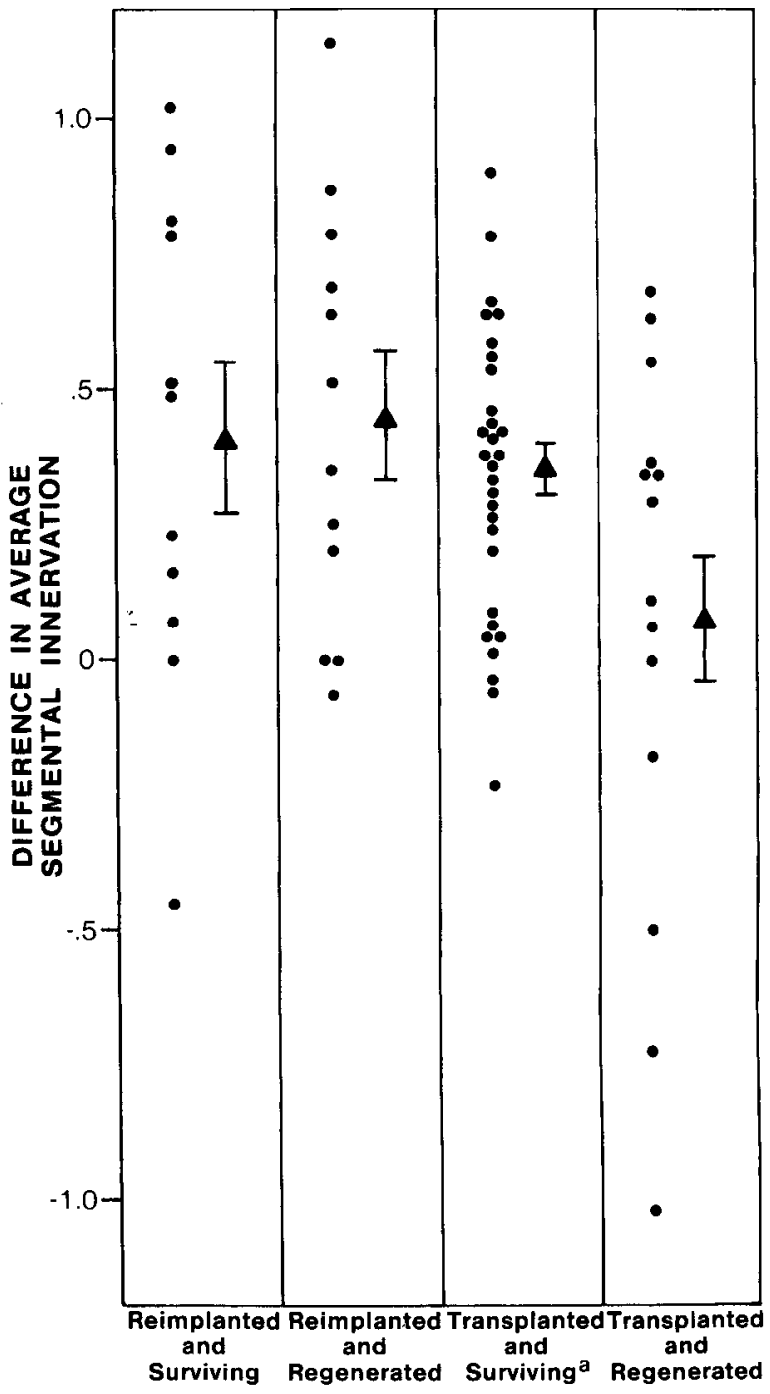

Figure 8. Difference in average segmental innervation between pairs of anterior and posterior muscles. Reimplanted and Surviving, data from 11 limbs in which combined anterior and posterior ILT muscles were removed and reimplanted in their original positions. Reimplanted and Regenerated, 12 limbs in which ILT muscles were surgically damaged and treated with bupivicaine. The muscles subsequently regenerated and were reinnervated in a new site. Transplanted and Survivinga, data from 30 limbs at 40-91 d after transplanting ILT muscles to the contralateral $\operatorname{limb}$, with the positions of the anterior and posterior heads exchanged ( ${ }^{\mathrm{a}}$ From Wigston, 1986). Transplanted and Regenerated, results from 14 limbs that were implanted with ILT muscles from the contralateral side and had been treated so that their myofibers would degenerate and subsequently regenerate. Muscles were reimplanted in reversed $a-p$ orientation, so that the original anterior ILT occupied the former site of the posterior ILT and vice versa.

ganglionic axons in the cervical sympathetic trunk. This may simply be because the moderate selectivity seen in the reinnervation of normal intercostal muscles transplanted to the neck became undetectable after its reduction by myofiber destruction and regeneration. An alternative is that the difference between their result and ours is a consequence of intrinsic differences in the 2 systems: mammalian autonomic preganglionic axons innervating segmented intercostal muscles, and urodele motor axons innervating limb muscles. Axolotl limbs will regenerate after amputation, and appear to regain their normal function, probably because individual muscles are reinnervated selec- 
tively (see Stephens and Holder, 1987). This remarkable capacity for regeneration may underlie the apparent difference between our result and that of Wigston and Sanes (1985).

Since the average difference in the segmental innervation of transplanted, regenerated anterior and posterior ILT muscles was not significantly different from zero $(0.08 \pm 0.12)$, one might argue that reinnervation was essentially random. However, the difference in average segmental innervation of muscles that regenerated in their original site was clearly different from zero $(0.45 \pm 0.12)$ and indistinguishable from the difference in average segmental innervation of muscles whose myofibers survived reimplantation to their original site $(0.41 \pm 0.14)$. Thus the relative positions of the anterior and posterior ILT must have an appreciable influence on the source of reinnervation. If all muscle recognition cues had, in fact, been abolished by myofiber degeneration, the difference between the average segmental innervation of a transplanted and regenerated ILT ought to have a negative value (recall that we express selectivity as the average segmental innervation of the original posterior ILT minus that of the original anterior ILT) and a magnitude similar to that seen in muscles that regenerate in their own site. However, muscles that regenerated in a new site after degeneration and transplantation were not reinnervated according to their new site (mean difference in average segmental innervation, 0.08 \pm 0.13 ). Rather, they were reinnervated in a pattern between that and the pattern of innervation they would have received had they been transplanted intact or reimplanted in situ. It follows that regenerated muscles must retain some of their original preference for particular axons, and therefore that some selectivity cues must survive myofiber regeneration. Our results are thus consistent with the existence of a set of muscle recognition cues that are not lost by myofiber destruction. Interestingly, a minor subset of the transplanted and regenerated muscles appeared to be reinnervated on the basis of their new position (see Fig. 8, bottom right). No other peculiarity of any kind was noted that might distinguish these muscles from the others. This occasional nonselective reinnervation, rather than a uniform, but small, reduction in selectivity might also underlie the more caudal reinnervation of the anterior ILT head of the transplanted and regenerated muscles (Fig. 7). We cannot explain why our treatment would completely abolish selectivity cues in some muscles, while allowing them to persist in others, but this would explain the observed differences in mean segmental innervation between transplanted, regenerated muscles and the other groups. This implies that there might be only one set of muscle recognition cues within a muscle, and that they are not usually eradicated by myofiber destruction. In either case, the existence of cues not associated with a muscle's original myofibers is implicated.

It is not clear where the cues that betray the original identity of a regenerated muscle originate. They might, for example, be inherited from satellite cells that survive the destruction of myofibers and proliferate to generate new muscle fibers. The selectivity cues could thus be passed onto a new myofiber generation in a lineage-dependent manner (Miller and Stockdale, 1986; Sanes, 1987). Another possibility is that some cues might survive myofiber degeneration because they are associated with other cell types within the muscle-for example, Schwann cells, endothelial cells, or even fibroblasts. Indeed, recent studies of developing chick limbs suggest that motoneuron axons innervate their target muscles as a result of guidance by cues associated with the connective tissue through which they travel (Dias and Lance-Jones, 1987), rather than according to the segmental origin of the myoblasts contributing to their target (Keynes et al., 1987). Alternatively, since molecules that mark synaptic sites have been found in the basal lamina surrounding muscle fibers (Sanes and Hall, 1979; Bayne et al., 1984; Fallon et al., 1985), it is conceivable that molecules of the extracellular matrix could distinguish individual muscles, thereby encouraging selective synapse formation between the muscles and certain motoneurons. These possibilities could be examined by eliminating other components of muscles and assaying the selectivity of muscle reinnervation.

\section{References}

Bayne, E. K., M. J. Anderson, and D. M. Fambrough (1984) Extracellular matrix organization in developing muscle: Correlation with acetylcholine receptor aggregates. J. Cell Biol. 99: 1486-1501.

Bennett, M., and N. Lavidis (1984) Development of the topographical projection of motor neurons to a rat muscle accompanies loss of polyneuronal innervation. J. Neurosci. 4: 2204-2212.

Benoit, P. W., and W. D. Belt (1970) Destruction and regeneration of skclctal muscle after treatment with a local anesthetic, bupivicaine (Marcaine). J. Anat. 107: 547-566.

Brown, M. C., and C. M. Booth (1983) Postnatal development of the adult pattern of motor axon distribution in rat muscle. Nature 304 : 741-742.

Clarke, P. G. H., and W. M. Cowan (1976) The development of the isthmo-optic tract in chick, with special reference to the occurrence and correction of developmental errors in the location and connections of isthmo-optic neurons. J. Comp. Neurol. 167: 143-164.

Dias, M., and C. Lance-Jones (1987) A possible role for somatopleural tissue in specific motoneuron guidance in the embryonic chick hindlimb. Soc. Neurosci. Abstr. 13:468.

Donahue, S. P., and A. W. English (1987) The role of synapse elimination in the establishment of neuromuscular compartments. Dev. Biol. 124: 481-489.

Donahue, S. P., and D. J. Wigston (1987) Specificity of muscle reinnervation in situ is not abolished by destroying muscle fibers. J. Cell Biol. 105: 64a.

English, A. W. (1986) Does synapse elimination shape neuromuscular compartments? Soc. Neurosci. Abstr. 12: 1118.

Fallon, J. R., R. M. Nitkin, N. E. Reist, B. G. Wallace, and U. J. McMahan (1985) Acetylcholine receptor-aggregating factor is similar to molecules concentrated at neuromuscular junctions. Nature 315: 571-574.

Farel, P. B., and S. E. Bemelmans (1985) Specificity of motoneuron projection patterns during development of the bullfrog tadpole (Rana catesbeiana). J. Comp. Neurol. 238: 128-134.

Holder, N., J. Mills, and D. A. Tonge (1982) Selective reinnervation of skeletal muscle in the newt Triturus cristatus. J. Physiol. (Lond.) 326: 371-384.

Keynes, R. J., R. V. Stirling, C. D. Stern, and D. Summerbell (1987) The specificity of motor innervation of the chick wing does not depend upon the segmental origin of muscles. Development 99: 565-575.

Lamb, A. H. (1976) The projection patterns of the ventral horn to the hind limb during development. Dev. Biol. 54: 82-99.

Lance-Jones, C. (1982) Motoneuron cell death in the developing lumbar spinal cord of the mouse. Dev. Brain Res. 4: 473-479.

Lance-Jones, C., and L. Landmesser (1980) Motoneurone projection patterns in the chick hind limb following early partial reversals of the spinal cord. J. Physiol. (Lond.) 302: 581-602.

Lance-Jones, C., and L. Landmesser (1981) Pathway selection by embryonic chick motoneurons in an experimentally altered environment. Proc. R. Soc. Lond. [Biol.] 214: 19-52.

Landmesser, L. (1978) The development of motor projection patterns in the chick hind limb. J. Physiol. (Lond.) 284: 391-414.

Mauro, A. (1961) Satellite cells of skeletal muscle fibers. J. Biophys. Biochem. Cytol. 9: 493-495.

McGrath, P. A., and M. R. Bennett (1979) The development of synaptic connections between different segmental motoneurons and striated muscles in an axolotl limb. Dev. Biol. 69: 133-145.

Miller, J. B., and F. E. Stockdale (1986) Developmental regulation of 
the multiple myogenic cell lineages of the avian embryo. J. Cell Biol. 103: 2197-2208.

O'Leary, D. D. M., and W. M. Cowan (1982) Further studies on the development of the isthmo-optic nucleus with special reference to the occurrence and fate of ectopic and ipsilaterally projecting neurons. J. Comp. Neurol. 212: 399-416.

O'Leary, D. D. M., J. W. Fawcett, and W. M. Cowan (1986) Topographic targeting errors in the retinocollicular projection and their climination by selective ganglion cell death. J. Neurosci. $\sigma: 3692-$ 3705 .

Oppenheim, R. W. (1981) Cell death of motoneurons in the chick embryo spinal cord. J. Neurosci. 1: 141-151.

Sanes, J. R. (1987) Cell lineage and the origin of muscle fibers. Trends Neurosci. 10: 219-221.

Sanes, J. R., and Z. W. Hall (1979) Antibodies that bind specifically to synaptic sites on muscle fiber basal lamina. J. Cell Biol. $83: 357-$ 370.

Stephens, N., and N. Holder (1987) Reformation of the pattern of neuromuscular connections in the regenerated axolotl limb. Development 99: 221-230.
Stephenson, R. S. (1979) Axon reflexes in axolotl limbs: Evidence that branched motor axons reinnervate muscles selectively. Exp. Neurol. 64: 174-189.

Weiss, P. (1936) Selectivity controlling the central-peripheral relations of the nervous system. Biol. Rev. 11: 494-531.

Wigston, D. J. (1986) Selective innervation of transplanted limb muscles by regenerating motor axons in the axolotl. J. Neurosci. 6: 27572763.

Wigston, D. J., and S. P. Donahue (1987) Cues associated with myofibers contribute to selective reinnervation of transplanted muscles. J. Cell Biol. 105: 64a.

Wigston, D. J., and P. R. Kennedy (1987) Selective reinnervation of transplanted muscles by their original motoneurons in the axolotl. J. Neurosci. 7: 1857-1865.

Wigston, D. J., and J. R. Sanes (1982) Selective reinnervation of adult mammalian muscle by axons from different segmental levels. Nature 299: 464-467.

Wigston, D. J., and J. R. Sanes (1985) Selective reinnervation of intercostal muscles transplanted from different segmental levels to a common site. J. Neurosci. 5: 1208-1221. 